Artículo original

\title{
DISEÑO E IMPLEMENTACIÓN DE UNA TARJETA ELECTRÓNICA DE EXPANSIÓN MODULAR BASADO EN PSOC PARA EL DESARROLLO DE APLICACIONES DE SISTEMAS EMBEBIDOS
}

\author{
DESIGN AND IMPLEMENTATION OF A PSOC-BASED \\ MODULAR ELECTRONIC EXPANSION CARD FOR THE \\ DEVELOPMENT OF APPLICATIONS OF EMBEDDED \\ SYSTEMS
}

Alberto Alvarado Fernández

Jorge Enrique Deza Concori Tito Leoncio Córdova Miranda

Información del artículo: Recibido: 18/06/2019. Aceptado: 29/06/2019

${ }^{1}$ Ingeniero Electrónico

${ }^{2}$ Magíster Docente de la Facultad de Ingeniería, Universidad Privada de Tacna 


\section{Resumen}

Los sistemas embebidos son aquellos que se encuentran en variedad de sistemas para aplicaciones específicas, donde tamaño, performance y tiempo de respuesta son prioridad. El presente informe muestra el diseño e implementación de una tarjeta de expansión modular, que permite desarrollar prototipos de sistemas embebidos de manera rápida empleando los periféricos diseñados en la tarjeta y las interfaces desarrolladas para el PSoC 5LP, siendo el desarrollo y comprobación de funcionamiento de la tarjeta de expansión modular el principal objetivo de la investigación. De acuerdo al diseño electrónico planteado de la tarjeta de expansión modular, como resultado se obtuvo flexibilidad en la etapa de prototipado de sistemas embebidos, así como se logró acelerar el desarrollo de prototipos de sistemas embebidos por medio del diseño de interfaces que verificaron además el buen funcionamiento de la tarjeta de expansión modular.

Palabras clave: Interfaces, Periféricos, PSoC 5LP.

\section{Abstract}

The city of Tacna is no stranger to the exposure of pollutants such as sedimentable particles that are generated due to stable weather conditions, urban growth, increased vehicular traffic, urban expansion, unpaved streets, among other factors. . Through the present research work that was carried out in the city of Tacna, it was possible to determine the concentration of sedimentable atmospheric dust in our city, for which 08 sampling stations were established where the Passive Sampling methodology was applied, which consisted of the placement of glass plates located on the upper level of homes and / or institutions, for a period of 04 months and at that time the information was collected every 30 calendar days. For the location of the stations to be sampled, the type of road, population density and traffic were taken into account; managing to determine with it 08 sampling zones located in the Center and Intermediate zone of the city of Tacna, taking as reference the civic walk of the city. The results obtained from the sampling of sedimentable particles indicate a final average value of $1.07 \mathrm{mg} / \mathrm{cm} 2 /$ month in all sampling stations, which is in the order of $0.57 \mathrm{mg} / \mathrm{cm} 2 /$ month above the guide values for atmospheric particles sediments of the World Health Organization - WHO, set at $0.50 \mathrm{mg} / \mathrm{cm} 2$ / month. According to the results obtained, it was possible to determine 3 points with a greater degree of significance, in which the PM2.5 and PM10 were monitored with the mobile station where the Automatic Monitoring methodology was applied.

Key Words: Air quality, particulate material

Julio - Diciembre del 2019.

\section{INTRODUCCIÓN}

La presente investigación se refiere al diseño electrónico de una tarjeta electrónica y sus interfaces, usando como microcontrolador un PSoC 5LP, de tal manera para desarrollar prototipos de sistemas embebidos.Un PSoC 5LP es un SoC diseñado, fabricado y distribuido por la empresa Cypress Semiconductor, siendo su principal característica el manejo de UDB (Universal Digital Block) que permite una amplia flexibilidad al momento de desarrollar prototipos. 
En la etapa de diseño se identificaron y delimitaron los requerimientos, así como los periféricos que deberán ir en el diseño electrónico. Se estudió de manera individual cada periférico y probo con el PSOC 5lp, corroborando su funcionamiento. El desarrollo de sistemas embebidos a lo largo de la historia ha ido evolucionando. El principal objetivo de un sistema embebido es que está diseñado para realizar una o algunas pocas funciones en tiempo real. En su desarrollo se deben trabajar dos ramas muy importantes, el desarrollo de hardware y de software. Los Microcontroladores comerciales necesitan de cierta electrónica para ser programados o para que funcionen. Uno de los principales inconvenientes que surgen a lo largo del diseño de hardware es su complejidad al momento de hacer funcionar los distintos periféricos como sensores, osciladores, programadores y para cada nuevo componente se debe añadir más y más conexiones electrónicas, llega un momento donde el diseño de hardware se hace excesivo para poder trabajar. Las nuevas tecnologías apuntan a un nuevo sistema: los SoC (System on Chip) estos nuevos dispositivos nos permiten tener posibilidades de diseño de hardware y diseño de software en un "software". Los dispositivos SoC (System on Chip) que integran más componentes internos que los microcontroladores actuales, ofreciendo mayor poder de procesamiento en empaquetados más pequeños, que permiten dispositivos más compactos y más baratos que antes. La placa de desarrollo PSoC 5LP es una de las últimas tarjetas desarrolladas por Cypress Semiconductor que ofrecen una solución para explorar y conocer los PSoC producidas por la misma compañía, pero carecen de periféricos externos para el desarrollo de proyectos, como sensores, actuadores e interfaces de comunicación. Definida las características y requerimientos del diseño, así como cumplir normas y estándares, será posible crear una tarjeta más accesible a estudiantes, comparado a las tarjetas de entrenamiento presentes en el mercado, un mayor número de periféricos permitirá desarrollar mayor número de proyectos; además a través de la producción de interfaces y aplicaciones, será posible mejorar la capacidad de desarrollo de proyectos de sistemas embebidos, así como disminuir su tiempo de desarrollo.

Ma, Li, Liu y Jin (2010), concluyen que no es ideal que solamente los estudiantes realicen teoría y práctica en un ambiente de laboratorio, también muestra el modelo de desarrollo de un módulo. En el desarrollo de una tarjeta de desarrollo para el PIC $18 f 4550$ (Alpaslan, Cem y Terzioglu, 2015), refieren que la metodología de desarrollo es probar cada uno de los módulos propuestos por separado, y luego a través de software de diseño ISIS Proteus, diseñar la tarjeta final con todos los módulos interconectados a un microcontrolador. Alpaslan et al. (2015) muestra ejemplos con la tarjeta de desarrollo para el PIC 18f4550, además que su metodología de diseño es similar como en la tarjeta de desarrollo para AVR (Ma et al. 2010). En el informe de prácticas desarrollada por Deza (2018), se da una introducción al manejo y uso de la tarjeta PSoC 5LP de la empresa Cypress Semiconductor, donde el PSoC posee bloques lógicos programables tanto en un microcontrolador como PLD's, así como bloques analógicos programables. Un sistema embebido "es un sistema electrónico que incluye un microcomputador, que es configurado para desempeñar una aplicación dedicada. El Software es programado en la ROM, no siendo accesible hacia el usuario, y solo resuelve una serie limitada de problemas, en los que el microordenador está integrado u oculto en el sistema." (Konstantakos, Chatzigeorgiou, Nikolaidis, Laopoulos, \& Member, 2008). "Es cualquier dispositivo que incluye una computadora programable pero no está hecha para ser una computadora de propósito general (Tiwari, n.d.) Un sistema embebido puede también ser un componente de algún otro sistema mucho mayor. Al momento de hablar de sistemas embebidos surge la confusión sobre los circuitos integrados, si bien un circuito integrado puede también cumplir una función en específica, la mayor ventaja de un sistema embebido es la flexibilidad que ofrece, ya que es mucho más sencillo modificar algunas líneas de código en el software que reemplazar todo el circuito integrado (Hardware). Los requisitos principales en este sistema son Disponibilidad, Fiabilidad, Confiabilidad, 
Seguridad, Bajo consumo, Capacidad de dar respuestas reactivas a eventos en un tiempo específico (Real time) y Tiempo de vida, que debe ser alrededor de 10 a 20 años. Su aplicabilidad puede darse en automovilismo, electrónica de consumo, trenes, aviación, telecomunicaciones, robótica, aplicaciones militares, telefonía móvil, sensores y conexiones ópticas.

\section{OBJETIVOS}

- Desarrollar periféricos para la tarjeta de expansión modular basada en el PSoC CY8C5888LTILP097 que permitan la flexibilidad de prototipado de sistemas embebidos.

- Desarrollar interfaces para los periféricos de la tarjeta de expansión con el software PSoC Creator que permitan comprobar el correcto funcionamiento de la tarjeta, así como disminuir el tiempo de desarrollo de prototipos de sistemas embebidos.

\section{METODOLOGIA}

Investigación aplicada cuasi-experimental, en el área de estudio de la Ingeniería electrónica y línea de investigación de diseño electrónico.

\section{1}

Al momento de diseñar la placa de entrenamiento modular se tuvo en claro los requerimientos de cada componente. Los periféricos se dividen en digitales, análogos y de comunicaciones. Se incluye en la figura 01 y 02 un esquemático general sobre los periféricos que se incluirá la tarjeta de entrenamiento.

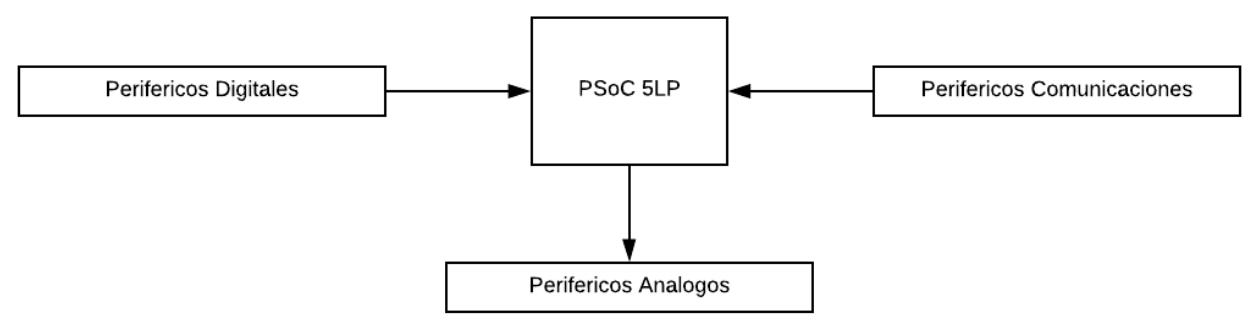

Fig.1. Diagrama de bloques de componentes en tarjeta

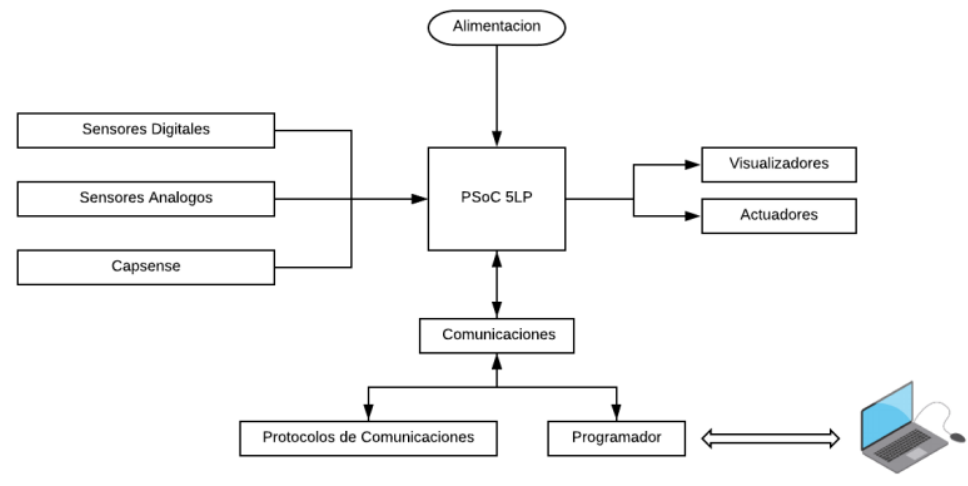

Figura 02. Diagrama de bloques de componentes expandido en tarjeta. 
Si bien se requiere realizar el mayor número de experiencias o interfaces con la placa PSoC 5LP, se nombrará los periféricos más comunes y más fáciles de encontrar en el mercado local (Tabla $1,2$ y 3$)$.

Tabla 11: Sensores digitales y análogos

\begin{tabular}{ll}
\hline \multicolumn{1}{c}{ Digitales } & Análogos \\
\hline Sensor de Humedad - DHT11 & LM35 \\
Pulsadores & Potenciómetro \\
Encoder Rotatorio & LDR \\
Push button & \\
Switch &
\end{tabular}

Tabla 2: Visualizadores y actuadores

\begin{tabular}{lc}
\hline \multicolumn{1}{c}{ Visualizadores } & Actuadores \\
\hline LCD 16x2 & Relé de estado sólido - SSR \\
Leds & \\
Led RGB & \\
Display 7 Segmentos, 4 digitos &
\end{tabular}

Tabla 3: Comunicaciones

\begin{tabular}{l}
\hline Comunicaciones \\
\hline Sensor Acelerómetro - MPU6050 (I2C) \\
Lector Micro SD - (SPI) \\
Sensor Bluetooth - HC05(UART) \\
Modulo Wi-fi \\
\hline
\end{tabular}

El PSoC para poder ser alimentado de manera externa, se debió retirar un diodo en el programador para que estos dos no alimenten a la vez. La extracción del D1 del kit mini prog se retira y se alimenta el PSoC con una fuente switching de $5 \mathrm{v}$. Para suplir las necesidades de un voltaje de $3.3 \mathrm{v}$ que es distinto a los $5 \mathrm{v}$ que se propone como fuente única de alimentación se usó reguladores AMS1117 3.3v para cada periférico con dicho nivel de alimentación. (Fig. 03

) Una vez idealizado, el bloque de los periféricos se procedió a diseñar el circuito de cada componente. Una vez que el diseño circuital de cada bloque se haya corroborado que funciona, se procedió a realizar el diseño de la PCB incluyendo pistas, pads, dimensionamiento, capas, artwork, serigrafía y fabricación. Una vez realizado el análisis y conocer los requerimientos de 
$\mathrm{E} / \mathrm{S}$, así como los periféricos a necesitar, se analizó a profundidad los componentes que integraran los periféricos, para determinar si es factible su integración en el proyecto. Cabe destacar que el diseño de librerías es propio, debido a que el programa Altium Designer no posee librerías de los componentes que se planea utilizar. El proceso de diseño de componentes se divide esquemático, footprint y modelo 3D. Se crearon las siguientes librerías para el diseño: PSoC 5 LP, LCD 16x2, Leds SMD y RGB, MPU6050, Switch Tactil SMD, Bluetooth HC 05, CD4050, ESP 8266 modelo 12E, Capacitores, Display 7 segmentos, Transistores SMD, DipSwitch, Encoder rotatorio, Sensores DHT y LDR, Relay Estado Solido, Buzzer, Potenciómetro, MicroSD, Capsense, Conectores y Headers, Terminales PCB. Ya conocido los fundamentos de los componentes por medio del diseño de librerías, se procede al diseño de periféricos; cada periférico realiza una función diferente tanto de entrada/salida y requiere de cierta cantidad de componentes discretos como resistencias, capacitores, leds, reguladores, etc., para su funcionamiento. Diseño PCB y ensamblaje.

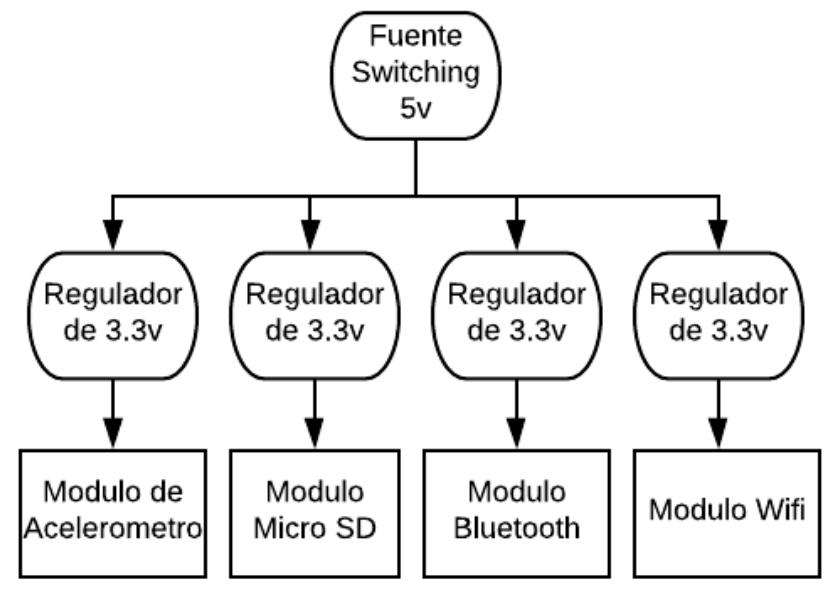

Figura 03. Diagrama de alimentación a periféricos.

Para obtener un archivo PCB Project donde se visualicen los componentes del esquemático, se utilizó el comando Update $\mathrm{PCB}$ en la pestaña Desig. Una vez que se presentan todas las conexiones en el esquemático y se exportan las netlist y footprints de los componentes a través de la actualización PCB, es necesario colocar todos los componentes en su sitio en el diseño PCB final. Cada capa en la etapa de diseño tiene un color, rojo para capa superior y azul para capa inferior. Una vez procedido con las pistas mas importantes, se puede aplicar autorouter y corregir si algunas pistas no estan de acuerdo al diseño. Para mejorar la respuesta en los pines del puerto analógico, así como funciones Capsense, se empleo el modelo en forma de rejilla y no sólida. Al termino del diseño, tenemos una $P C B$ de dimensiones $17 \times 17 \mathrm{~cm}$, según Altium Designer, y el modelo 3D que indicara que todas los componentes tienen el espaciado necesario.

\section{RESULTADOS}


Para verificar el correcto funcionamiento de la PCB, se empleó las aplicaciones creadas en PSoC Creator. Es en este punto donde se verifica el correcto funcionamiento de los componentes del hardware diseñado en Altium Designer. Como primer paso, se enciende el módulo a través del conector jack 5V DC. (Fig. 04)

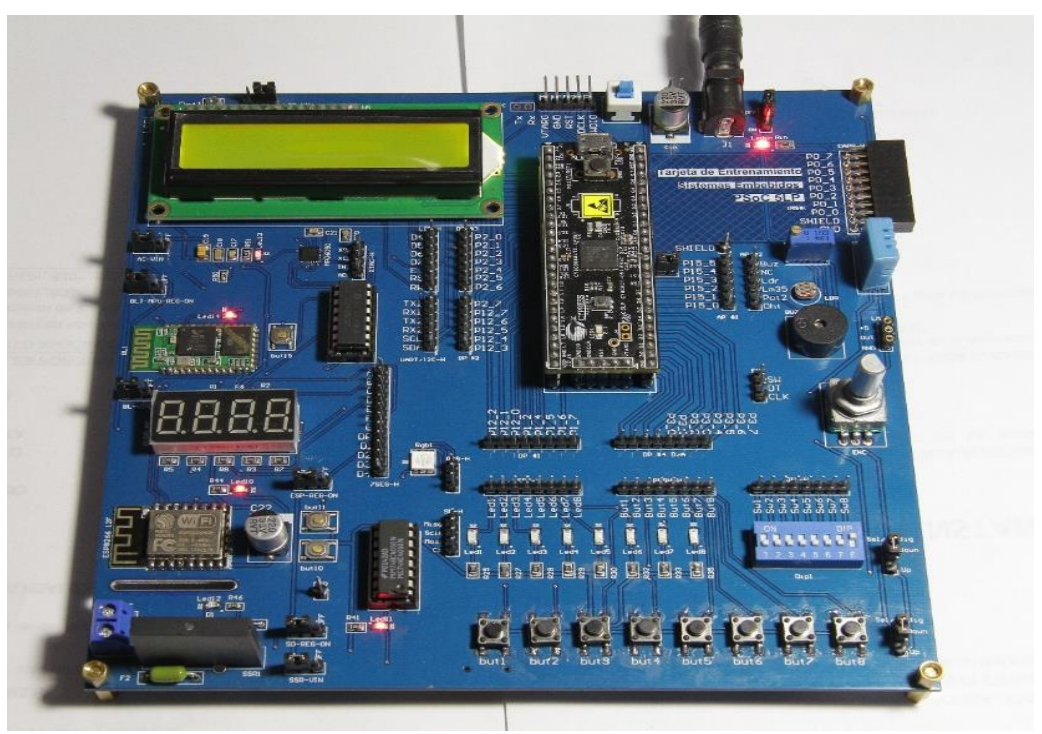

Figura 4: Encendido de tarjeta de expansión modular para PSoC 5LP

La primera comprobación es el voltaje presenten en los diferente componentes y módulos de la PCB. Se comprueba mediante multímetro que no exista caída de voltaje hacia el pin VDD del PSoC 5 LP, así como a los componentes LCD, SSR, CD4050, componentes analógicos.

Además, se tiene que comprobar la alimentación de 3.3v hacia los componentes Bluetooth, ESP8266, Acelerómetro y salida exterior del puerto analógico, verificado tal, se procede a comprobar que los reguladores de $3.3 \mathrm{v}$ no presentan sobrecalentamiento, al no encontrar problemas ni excesiva temperatura, se procede a realizar la misma comprobación con los 5 PCB diseñadas y fabricadas. La comprobación de leds y botones se realiza mediante aplicación 4, que prueba las configuraciones pull-up y pulldown de los botones, o dipswitch, mientras que las salidas conectadas a los leds, encendían o apagan éstos últimos. Otra manera más práctica de comprobar el funcionamiento de los leds es a través de del uso del multímetro o aplicación de voltaje de 5 v. (Fig 5)

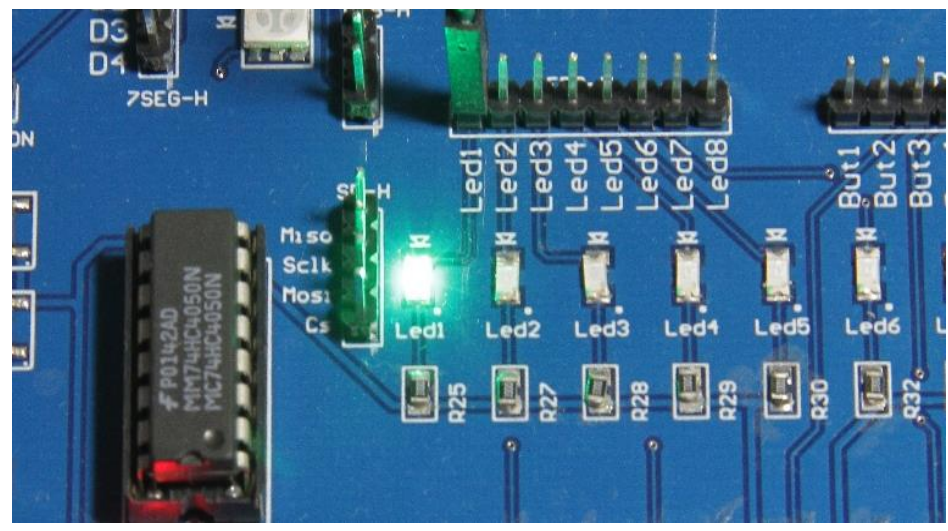

Figura 5:2 Comprobación de Leds SMD. 
Para comprobar el LCD; se carga el programa de la aplicación hacia el PSoC, mostrando el mensaje a "Prueba de LCD", en la siguiente imagen se visualiza el funcionamiento del mensaje en módulo LCD. (Fig 6)

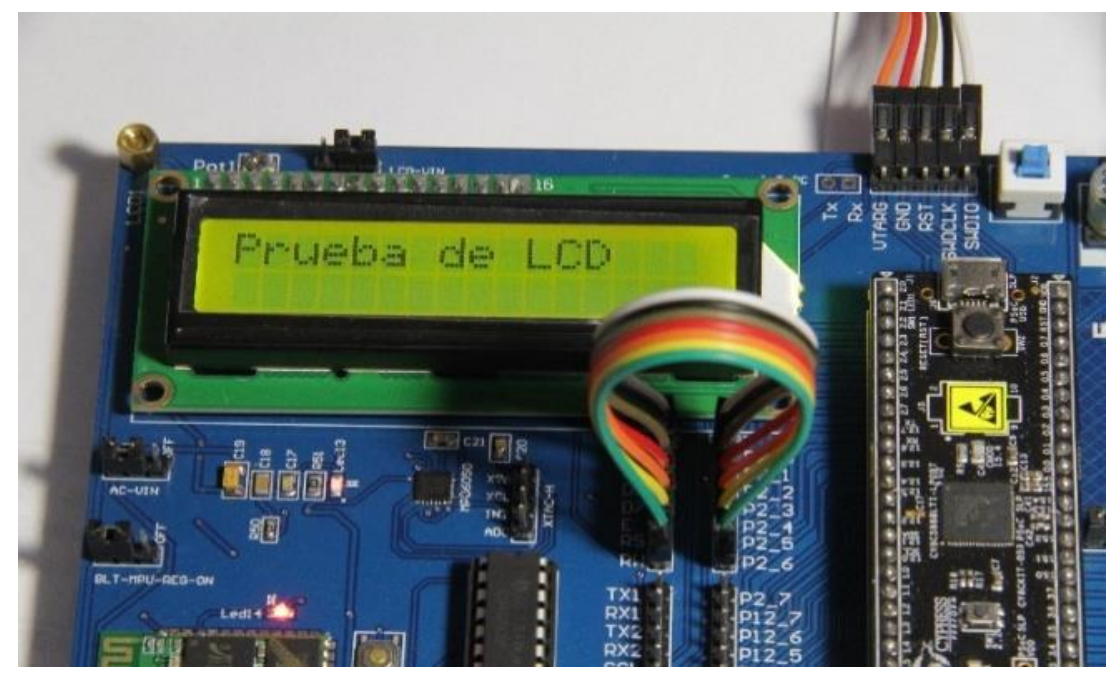

Figura 6: Comprobación del LCD en tarjeta PCB

Para la comprobación del módulo Bluetooth, se realiza el vínculo entre el módulo con el celular y abrir la aplicación, para finalmente enviar datos seriales desde el celular al PSoC $5 \mathrm{LP}$. El envío de datos seriales corrobora el funcionamiento del integrado CD4050B. (Fig. 7)

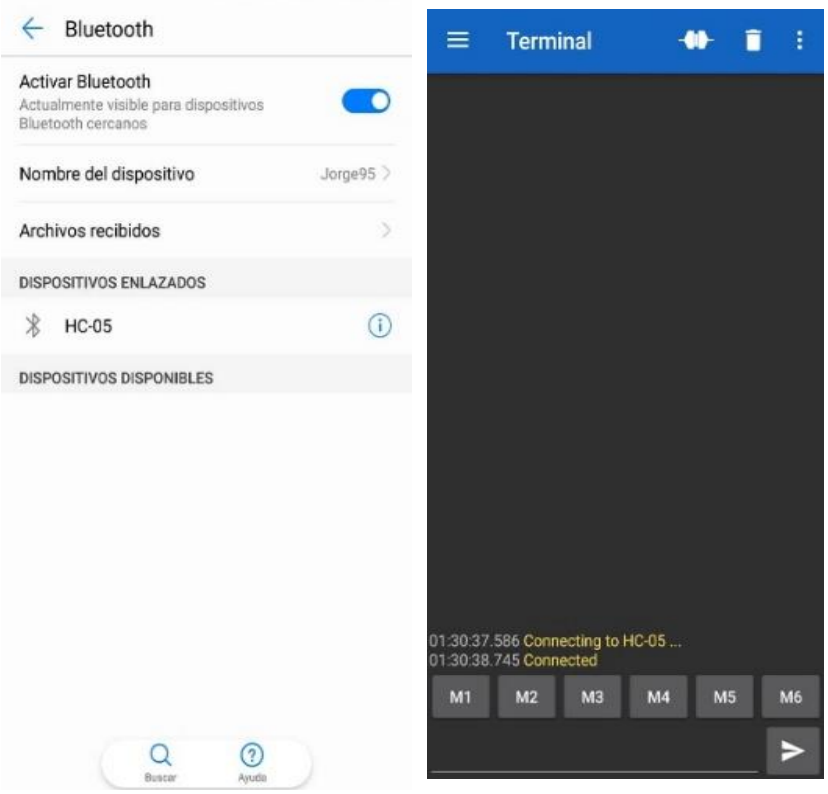

Figura 7: Conexión de modulo Bluetooth con celular.

Para la comprobación del módulo ESP8266, se enciende el módulo y se carga el programa como se mencionó en el capítulo anterior, revisando la red local, se introduce la IP del módulo Wifi en 
el buscador web Chrome, para verificar que se muestra una página web que muestra un dato proveniente de la tarjeta PSoC 5 LP. (Fig. 8)

\section{Data from Serial to Esp8266 12F Web Server}

Data:

10

Figura 8: Envió de datos seriales a esp8266, mostrado en página web

Para la comprobación del módulo encoder, módulo display 7 segmentos, se siguió la aplicación 3 , donde el número de rotaciones del encoder es contado por el PSoC 5 LP y mostrando el resultado en el display 7 segmentos. (Fig.9)

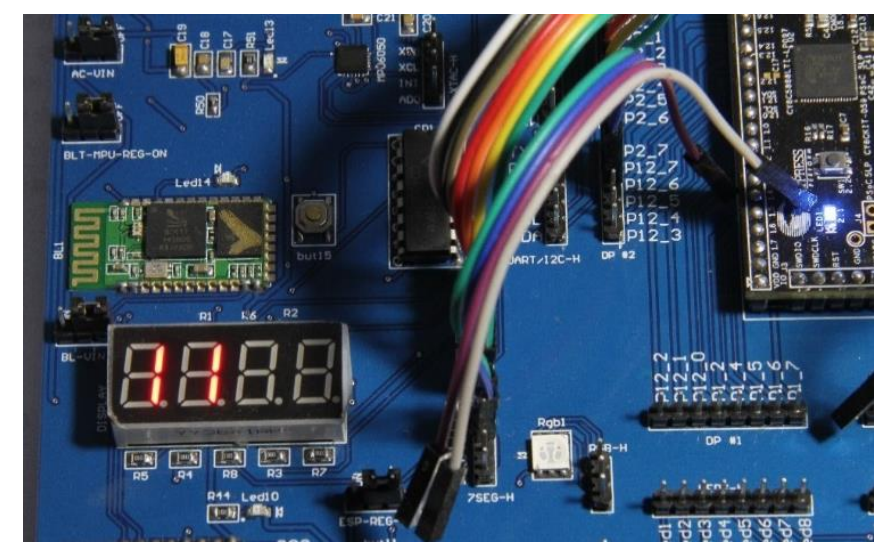

Figura 9 segmentos contando rotaciones de encoder.

El componente MPU 6050 se puede comprobar su correcto funcionamiento al emplear el programa Bridge control, que permite usar la comunicación I2C a USB del programador KitProg, identificando de manera satisfactoria el acelerómetro mediante este software. La dirección del acelerómetro es D0 68, de acuerdo a datasheet. (Fig 10)

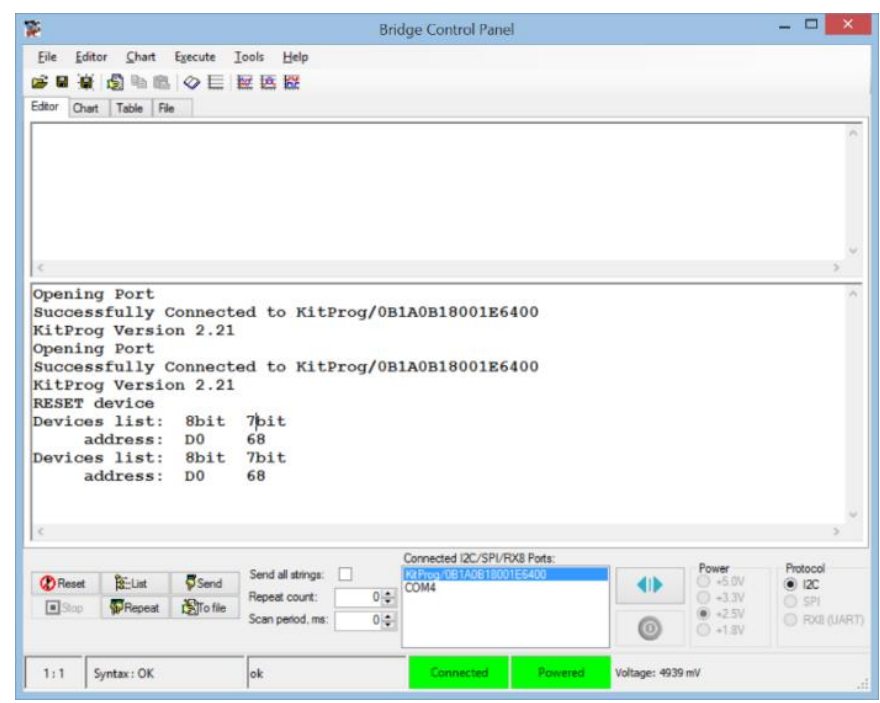


Figura 10: Identificación de Módulo MPU6050 en Bridge Control.

Para comprobar el funcionamiento de los sensores analógicos como potenciómetro y LDR, se siguió la aplicación 6 , a través del uso de un ADC, se leen los valores de los sensores y se envían a un puerto serie hacia la PC. (Fig. 11)

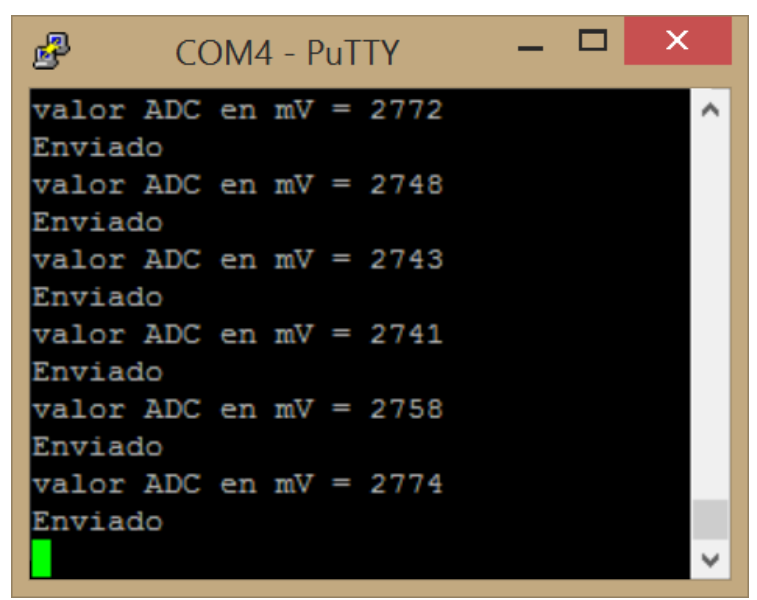

Figura 3: Lectura de sensores analógicos vía puerto Serie.

Para la comprobación del Led RGB, solo fue necesario llevar los pines a alimentación de 5V, así como el módulo buzzer, que, con la resistencia empleada, produce un sonido a bajo volumen. Una vez comprobado todos los periféricos en todas las placas PCB, se da la conformidad de su correcto funcionamiento.

\section{CONCLUSIONES}

Mediante el método de diseño e implementación que incluye el análisis, requerimientos del circuito, etapa de prototipado, uso software de diseño electrónico, fabricación y ensamblaje; se ha logrado la integración de diferentes periféricos digitales (LCD, Display, Relay, SD, Leds, Switches, Encoder, DHT y Acelerómetro), analógicos (Potenciómetro, LDR, Buzzer, Capsense) e inalámbricos (Wifi y Bluetooth), en una tarjeta de expansión modular diseñada para el PSoC 5LP, permitiendo la flexibilidad en la etapa de prototipado de un sistema embebido.

Se logró realizar aplicaciones que permiten usar y comprobar el correcto funcionamiento de los periféricos mediante las interfaces diseñadas en PSoC Creator, evaluando la documentación de las hojas técnicas dadas por el fabricante, la reutilización de éstas logra disminuir el tiempo de desarrollo de un prototipo para un sistema embebido, además de conocer las posibles configuraciones de la tarjeta de expansión modular.

\section{RECOMENDACIONES}


Es posible desarrollar a futuro una tarjeta de expansión modular sin la necesidad de emplear un kit de desarrollo como el PSOC 5 LP, sino usando solamente el PSoC integrado directamente en la PCB. Debido a la flexibilidad de conexión de la tarjeta de expansión modular, se permite usar otro hardware con una tarjeta PCB de adaptación de pines adecuada.

Queda pendiente el desarrollo de aplicaciones más complejas para conocer a profundidad la funcionalidad de los periféricos y del PSoC 5LP, debido a que es un sistema programable de alto nivel de recursos para sistemas embebidos. La tarjeta de expansión modular es una herramienta que puede ser utilizada para el desarrollo de futuros prototipos en trabajos de tesis relacionado a sistemas embebidos.

\section{REFERENCIAS BIBLIOGRÁFICAS}

FATIH A., ABDULLAH C., HAKAN T. (2015) The design of a Test \& Development Board for the Training of PIC18F4550 Microcontroller. 2nd International Conference on Information Science and Control Engineering.

CYPRESS SEMICONDUCTOR. PSoC Creator User Guide. San Jose, CA 95134-1709.

JULIÁN R., CÉSAR P. Introducción al PSoC5LP Teoría y aplicaciones prácticas. Universidad Distrital Francisco José de Caldas. Manizales, Caldas, Colombia.

JORGE DEZA C. Informe de prácticas pre-profesionales en los laboratorios de la escuela profesional de ingeniería electrónica. Universidad Privada de Tacna, Tacna.

FRANCISCO C., CRISTIAN V., ALEJANDRO A. Diseño y fabricación de circuitos impresos con Altium Designer. $-2^{a}$ ed. Buenos Aires.

MATT D. Porting the Arduino Library to the Cypress PSoC in PSoC Creator. November $11,2011$.

MARCO D., Mónica P. Diseño de un entrenador de microcontroladores PIC para el laboratorio de microcontroladores del Instituto Universitario Jesús Obrero. Universidad Simón Bolívar. Sartenejas, Caracas, Miranda, Venezuela

PSoC CHILE. Santiago de Chile, Chile. https://psoc-chile.com/acerca-de/psoc/

Iván Cuadros Acosta. (10 de enero 2017). Geek Electrónica.: https://geekelectronica.com/quees-psoc/

Chao M., Qingli L., Zhongyuan L, y Yu J.(2010), Low cost AVR microcontroller development kit for undergraduate laboratory and take-home pedagogies,"

BusinessWire (2017) Top 7 Vendors in the Global Microcontrollers Market from 2017-2021: Technavio. Extraído de : https://www.businesswire.com

Marco Chiappetta (2013) ARMs Race: Licensing vs. Manufacturing In Mobile. Extraido de: https://hothardware.com

Altium (2017). Connectivity and Multi-Sheet Design. Extraído de: https://techdocs.altium.com

ARM (s.f). Why ARM CPU Architecture. Extraido de: https://www.arm.com

Ingeniería investiga Vol. 1 (1) Julio - diciembre 2019. 
Electronic Schematics- Need to Know (s.f). Extraido de https://www.build-electroniccircuits.com

Bai, Y. (2016) 'Practical Microcontroller Engineering with ARM ${ }^{\circledR}$ Technology'.

Joseph Y.(201) The Definitive Guide to ARM Cortex M3 and Cortex M4 Processors

Jiacun Wang (2017) Real-Time Embedded Systems.

Robert Ashby (2005) Designers Guide to Cypress PSOC Robert Ashby

ZEGARRA, J. (2014). Diseño de un contador en VHDL para la reducción del margen de error en el conteo de pulsos del sensor de detector de cinta adhesiva. (Tesis de grado). Universidad Privada de Tacna. Tacna.

GONZALES, K., MENDEZ, J. (2011). Diseño y fabricación de un módulo entrenador implementando la familia de microcontroladores dsPIC33F. (Tesis de grado). Universidad Pontificia Bolivariana. Bucaramanga.

LINO, A. (2014). Diseño e implementación de plataforma basada en microcontroladores PIC para facilitar el estudio practico y la elaboración de proyectos en la carrera de ingeniería en telecomunicaciones. (Tesis de grado). Universidad católica de Santiago de Guayaquil. Guayaquil.

CYPRESS SEMICONDUCTOR (2017). PSoC 5LP Architecture Technical Reference Manual. San Jose,CA 9513-1709, California. 